\title{
Au Nanoclusters Ameliorate Shigella Infectious Colitis by Inducing Oxidative Stress
}

\author{
Xiaoxiao $\mathrm{Wu}^{1}{ }^{1, *}$ \\ Yongyan Chen $\mathbb{D}^{1, *}$ \\ Yangheng Zhang ${ }^{2} *$ * \\ Yunjie Shan' \\ Zhiyue Peng' \\ Bing $\mathrm{Gu}^{1,3}$ \\ Huan Yang $\mathbb{D}^{\prime}$ \\ 'Xuzhou Key Laboratory of Laboratory \\ Diagnostics, School of Medical \\ Technology, Xuzhou Medical University, \\ Xuzhou, 22 1004, People's Republic of \\ China; ${ }^{2}$ Department of Periodontology, \\ Nanjing Stomatological Hospital of \\ Nanjing University School of Medicine, \\ Nanjing, 210008, People's Republic of \\ China; ${ }^{3}$ Laboratory Medicine, Guangdong \\ Provincial People's Hospital, Guangdong \\ Academy of Medical Sciences, \\ Guangzhou, Guangdong, 510000 , People's \\ Republic of China
}

*These authors contributed equally to this work
Correspondence: Huan Yang; Bing Gu Email yanghuan2015@tmu.edu.cn; gb20031129@163.com
Background: Shigella infection has always been a global burden, and it particularly threatens children between the ages of 1 and 5 years. Economically underdeveloped countries are dominated by Shigella flexneri infection. The most effective method to treat Shigella is antibiotics, but with the abuse of antibiotics and the prevalence of multidrug resistance, we urgently need a relatively safe non-antibiotic treatment to replace it. Ultrasmall Au nanoclusters (NCs) have special physical and chemical properties and can better interact with and be internalized by bacteria to disrupt the metabolic balance. The purpose of this study was to explore whether Au NCs may be a substitute for antibiotics to treat Shigella infections.

Methods: Au NCs and Shigella Sf301, R2448, and RII-1 were cocultured in vitro to evaluate the bactericidal ability of Au NCs. The degree of damage and mode of action of $\mathrm{Au}$ NCs in Shigella were clearly observed in images of scanning electron microscopy (SEM). In vivo experiments were conducted to observe the changes in body weight, clinical disease activity index (DAI) and colon (including length and histopathological sections) of mice treated with $\mathrm{Au}$ NCs. The effect of Au NCs was analysed by measuring the content of lipocalin-2 (LCN2) and Shigella in faeces. Next, the changes in Shigella biofilm activity, the release of reactive oxygen species (ROS), the changes in metabolism-related and membranerelated genes, and the effect of $\mathrm{Au}$ NCs on the body weight of mice were determined to further analyse the mechanism of action and effect.

Results: $\mathrm{Au}$ NCs $(100 \mu \mathrm{M})$ interfered with oxidative metabolism genes, induced a substantial increase in ROS levels, interacted with the cell membrane to destroy it, significantly killed Shigella, and effectively alleviated the intestinal damage caused by Shigella in mice. The activity of the biofilm formed by Shigella was reduced.

Conclusion: The effective antibacterial effect and good safety suggest that $\mathrm{Au}$ NCs represent a good potential alternative to antibiotics to treat Shigella infections.

Keywords: Shigella, Au nanoclusters, antibacterial effect, reactive oxygen species, oxidative stress

\section{Introduction}

Shigella is a facultative anaerobic gram-negative bacilli without flagella and with fimbriae. Kiyoshi Shiga confirmed that it was the culprit causing bacillary dysentery in $1897 .{ }^{1}$ Since then, Shigella has become the main pathogen causing bacillary dysentery worldwide. A retrospective investigation of cases from 1966 to 1997 by Kotloff et al showed that among 164.7 million cases, more than 99\% occurred in developing countries, and $80 \%$ of them occurred in Asia. The review by Bardhan et al concluded that the number of cases in Asia was still high, with approximately 125 million cases per year from 1990 to 2009.,3 Most importantly, bacillary dysentery is more harmful to children and is one of the main causes of death in 
children. ${ }^{4,5}$ It is also the most widely distributed intestinal infectious disease with the highest incidence in China.

Shigella is divided into four serotypes, namely, Shigella dysenteriae, Shigella flexneri (S. flexneri), Shigella baumannii and Shigella sonnei. Bacterial dysentery is mainly caused by $S$. flexneri, especially in developing countries and countries with low economic levels. This disease is related to health, the environment, resources and other factors. ${ }^{6,7}$ Faecal-oral transmission is the most important transmission mode of Shigella, ${ }^{8}$ while invasiveness and endotoxin are the pathogenic factors of Shigella. Shigella adapts to gastric acid; ${ }^{9}$ the bacteria pass through the stomach and small intestine and finally reach the large intestine to promote infection. Endotoxin increases the permeability of the intestinal mucosa and destroys it. ${ }^{10}$ In addition, Shigella enters the intestinal epithelium through microfolded cells, rapidly induces the death of macrophages, and spreads to neighbouring cells under the cover of actin. ${ }^{11,12}$ The NF-kB pathway activated by the chlamydial type III secretion apparatus upregulates inflammatory factors, ${ }^{13}$ initiates neutrophil infiltration and destroys the integrity of intestinal epithelial cells, allowing more Shigella to easily enter the submucosa and produce an infection. ${ }^{11,14}$ In addition, Shigella changes the composition of connexins, weakening the sealing of endothelial cells, which aggravates the inflammatory response ${ }^{15}$ and ultimately manifests as watery diarrhoea, mucus pus and blood in the stool and other inflammatory reactions. Moreover, Shigella can cause invasive infections such as meningitis and osteomyelitis. ${ }^{16,17}$ In young children, intussusception or necrotizing enterocolitis is a common infection. ${ }^{18}$ Currently, the most reliable treatment for these bacteria is antibiotics. In 2017, the World Health Organization selected ciprofloxacin as the first choice for treatment. However, with the evolution and mutation of the pathogen, multidrug resistance has become a stumbling block to Shigella treatment. Multidrug resistance poses a great threat to most special populations with low immunity and even contributes to Shigella infection. ${ }^{19-}$ ${ }^{21}$ Scientists have gradually shifted the treatment of Shigella infections to other areas. Probiotics are also the focus of research. ${ }^{22}$ Short-chain fatty acids have been shown to assist therapy, ${ }^{23}$ and bacteriophages have also suggested as a treatment, ${ }^{24}$ but longer follow-up of the treatment is required. Many ethical guidelines restrict vaccine development, and no licenced vaccines have been developed. ${ }^{25}$ Therefore, a new treatment for Shigella with more promise is needed.
Nanoparticles have become a hot topic in medicine and biology. Using various technologies, such as electrostatic bonding and physical adsorption, nanoparticles are coupled with different ligands and biomolecules to allow them to exert different effects. ${ }^{26}$ The detection of $\mathrm{Au}$ nanoclusters (Au NCs) relies on surface plasmon resonance (SPR), which specifically marks and quantifies macromolecules under the microscope, and Au NCs have an important auxiliary role in the treatment of diseases. ${ }^{27}$ In addition, lateral flow assays using labelled $\mathrm{Au}$ NCs and biosensors for their applications can also be applied to the detection of Shigella. ${ }^{28,29}$ In the treatment of diseases, Au NCs can be used as drug carriers to specifically target and attack target cells. Au NCs have proven to be promising delivery vectors in the treatment of bacterial infections and tumours, ${ }^{30,31}$ such as Staphylococcus aureus infections. ${ }^{32}$ More importantly, Au NCs are a "drug" with great potential. After being modified by small molecules, they exhibit a high specific surface area and high surface chemical activity. Au NCs attack bacterial membranes and interfere with cellular metabolism, effectively killing many gram-negative bacteria and gram-positive bacteria, such as Staphylococcus epidermidis, Bacillus subtilis, Escherichia coli, Pseudomonas aeruginosa. ${ }^{33,34} \mathrm{Au} \mathrm{NCs}$ also show significant antifungal activity toward some fungi, such as Aspergillus and Penicillium. Due to the membrane structure, $\mathrm{Au} \mathrm{NCs}$ will be more effective against gram-negative bacteria. ${ }^{37}$ In addition, gold is a stable inert metal, and $\mathrm{Au}$ NCs have been proven to display good biocompatibility, low cytotoxicity, and considerable safety. ${ }^{34-36}$ We have previously proven that Au NCs effectively kill Clostridium difficile, ${ }^{36}$ but their function in killing Shigella remains to be studied.

Shigella infectious dysentery has become a global burden that cannot be ignored. With the prevalence of antibiotic abuse, Shigella resistance has become increasingly intense. This study explored the bactericidal effect of $\mathrm{Au}$ NCs on Shigella for the first time. At the same time, experiments in mice were carried out to further prove and explain the antibacterial effect of Au NCs and explore the possible mechanism underlying the antibacterial effect of $\mathrm{Au}$ NCs. In summary, Au NCs are very likely to become the focus as the next treatment for Shigella infection.

\section{Materials and Methods}

\section{Synthesis and Modification of Au NCs}

First, $0.25 \mathrm{~mL}$ of hydrogen tetrachloroaurate (III) hydrate $\left(\mathrm{HAuCl}_{4}\right)(20 \mathrm{mM}), 1 \mathrm{~mL}$ of $10 \mathrm{mM} \mathrm{6-mercaptohexanoic}$ 
acid (MHA) and $3.35 \mathrm{~mL}$ water were mixed and stirred to obtain an Au-MHA complex. Then, $0.3 \mathrm{~mL}$ of $\mathrm{NaOH}$ (1 $\mathrm{M}$ ) was added to dissolve it, and $0.1 \mathrm{~mL}$ of a newly prepared $112 \mathrm{mM} \mathrm{NaBH}$ solution was added. After $3 \mathrm{~h}$, the antigen was collected and treated with stirred cells (model 8010, Millipore Corporation, USA) by ultrafiltration. The size and quality of Au NCs were characterized using transmission electron microscopy (TEM) (JEM2100, Japan), dynamic light scattering (DLS) (Malvern Zetasizer Nano ZS90, UK) and ultraviolet-visible (UVVis) spectrometry (Shimadzu, UV-3600, Japan). Au NCs modified with MHA ligands were finally obtained. ${ }^{36}$

\section{Growth and Culture of Strains}

The Shigella strain used in this study was donated by $\mathrm{Xu}^{38}$ as Shigella Sf301-gfp (ampicillin screening marker). In this study, $S$. flexneri was stored at $-80^{\circ} \mathrm{C}$ and resuscitated before use. The standard strain Sf301 and clinically resistant strains R2448 and RII-1 (the results of the susceptibility analysis of strains R2448 and RII-1 are shown in Tables S1 and S2) were grown in trypticase (tryptic) soy broth (TSB) (Aoboxing, Beijing, China) and cultured at $37^{\circ} \mathrm{C}$ for $24 \mathrm{~h}$. Sf301-GFP was grown in TSB medium containing $100 \mu \mathrm{g} / \mathrm{mL}$ ampicillin and cultured at $37^{\circ} \mathrm{C}$ for $24 \mathrm{~h}$.

\section{Detection of the Antibacterial Activity of Au NCs Toward Strains Sf30I, R2448, and RII-I}

We conducted antibacterial tests on the three strains (Sf301, R2448 and RII-1) to determine whether Au NCs had antibacterial activity against Shigella. The bacteria were cultured in TSB liquid medium at $37^{\circ} \mathrm{C}$ for up to $24 \mathrm{~h}$ and then added to bacterial culture tubes with $\mathrm{Au} \mathrm{NC}$ concentrations of 0,50 and $100 \mu \mathrm{M}$. Sf301 was initially incubated with AuNCs for $1 \mathrm{~h}$ and $2 \mathrm{~h}$, and R2448 and RII-1 were incubated for $2 \mathrm{~h}$. Then, cultures were incubated for $48 \mathrm{~h}$ at $37^{\circ} \mathrm{C}$ in an anaerobic environment. Finally, the cfu of Shigella was counted, and the bacterial growth curve was plotted.

\section{Scanning Electron Microscopy (SEM) Characterization of Sf30I}

Sf301 was cocultured with $0 \mu \mathrm{M}$ and $100 \mu \mathrm{M}$ Au NCs in the dark for $2 \mathrm{~h}$, and then the supernatant was collected by centrifugation and the pellet was washed twice with normal saline. After adding $100 \mu \mathrm{L}$ of fixative solution, it was placed at $4^{\circ} \mathrm{C}$ for $12 \mathrm{~h}$, washed with $0.1 \%$ phosphate- buffered saline (PBS) (3 times, $10 \mathrm{~min}$ each), and then dehydrated with $25 \%$ ethanol $/ 50 \%$ ethanol $/ 70 \%$ ethanol/ $80 \%$ ethanol $/ 90 \%$ ethanol $/ 95 \%$ ethanol (2 times, $15 \mathrm{~min}$ each). The film was then dried, coated, observed and photographed under an SEM (Hitachi-S4800, Japan).

\section{Induction of S. flexneri Enteritis and Administration of Au NCs}

Male C57BL/6J mice (16-18 g) were purchased from Zhejiang Vital River Company. All animal experiments were performed according to the standards in the Guide for the Care and Use of Laboratory Animals published in 2011 (Institute of Laboratory Animal Resources of National Research Council, United States). ${ }^{39}$ All mouse studies were evaluated by the Laboratory Animal Ethics Committee of Xuzhou Medical University (IACUC number: 202012A200), Xuzhou, China. The room temperature was $25^{\circ} \mathrm{C}$, and the humidity was $40-70 \%$. The mice were housed on a $12 \mathrm{~h}$ alternating day and night cycle, and standard laboratory sterilized feed and water were freely available.

The mice were divided into the normal control group (NC), S. flexneri infection group (SF) and Au NC treatment group (SF-Au NCs), with 10 mice in each group. First, the SF group and the SF-Au NC group were pretreated for 7 days. Mice in the SF group were gavaged with $200 \mu \mathrm{L}$ of sterile water for 7 days, and mice in the SF-Au NC group were gavaged with $200 \mu \mathrm{L}$ of $\mathrm{Au}$ NCs $(200 \mu \mathrm{M})$ for 7 days. After pretreatment, mice were provided antibiotic-containing drinking water for 3 days. The antibiotic concentrations in drinking water were as follows: metronidazole $215 \mathrm{mg} / \mathrm{L}$, colistin $850 \mu \mathrm{g} / \mathrm{mL}$, gentamicin $35 \mathrm{mg} / \mathrm{L}$, and vancomycin $45 \mathrm{mg} / \mathrm{L}$. During the 4 days, mice in the SF group and SF-Au NC group were treated with the same treatment as the pretreatment. Then, mice in the SF and SF-Au NC groups were gavaged with $5 \times 10^{8}$ CFU S. flexneri on day 0 , followed by continuous gavage with $200 \mu \mathrm{L}$ of sterile water or $200 \mu \mathrm{M}$ Au NCs for 3 days, respectively. During the entire post infection period, clinical scores were recorded for each mouse. The mice were euthanized on day 3 , and the colon length of the mice was observed and measured. The faeces were collected and frozen at $-80^{\circ} \mathrm{C}$.

\section{Colon Histological Score}

The distal colon tissue of the mouse was fixed with a $4 \%$ paraformaldehyde solution and sent to Wuhan Servicebio Technology Co., Ltd. (Wuhan, China) to prepare 
haematoxylin-eosin (H\&E)-stained sections. The lesions of the colon tissue were observed under a microscope and scored according to the scoring criteria listed in Table S3. Histological injury score = inflammatory infiltration + crypt damage + crypt damage degree + the absence of oedema.

\section{Detection of the Faecal Lipocalin-2 (LCN2) Content}

When the mice showed symptoms of infection, such as weight loss and loose stools, the mouse faeces were collected and stored at $-80^{\circ} \mathrm{C}$. Before testing, the supernatant was collected after homogenization, and the LCN2 content in faeces was detected using the protocol of the LCN2 ELISA Kit (Biotyscience) to assess the level of colitis in mice.

\section{Measurement of the Tissue Bacterial Load}

The collected faeces were weighed, sterile PBS was added to prepare a homogenate, and the bacteria were counted using the plate counting method.

\section{Detection of Sf30I Biofilm Activity}

A turbidimetric metre (DensiCHEK ${ }^{\mathrm{TM}}$ Plus, USA) was used to configure a 0.5 McKenzie concentration bacterial solution that was co-cultured with 0 or $100 \mu \mathrm{M}$ Au NCs in a 96-well plate at $37^{\circ} \mathrm{C}$ for $24 \mathrm{~h}$. The samples were centrifuged, the supernatant was discarded, and the pellet was washed 3 times with PBS. One hundred microliters of MTT were added, incubated for $30 \mathrm{~min}$ before DMSO (100 $\mu \mathrm{L} /$ well) was added, and then the absorbance was measured at $570 \mathrm{~nm}$ using the $\mathrm{iMark}^{\mathrm{TM}}$ microplate reader (Bio-Rad, USA) to determine the OD value.

\section{Detection of ROS Release from Sf30I}

One millilitre of $5 \times 10^{8} \mathrm{CFU} / \mathrm{mL}$ bacterial solution was added to two Eppendorf (EP) tubes, centrifuged, and the supernatant was discarded. One millilitre of normal saline was added to each tube, along with Au NCs or the same amount of normal saline. The samples were mixed, and $400 \mu \mathrm{L}$ of each mixture were incubated in the dark at $37^{\circ} \mathrm{C}$ for $2 \mathrm{~h}$. Then, $20 \mu \mathrm{L}$ of DCFH-DA $(8 \mathrm{mg} / \mathrm{mL})$ were added in the dark, and incubated for $30 \mathrm{~min}$ in the dark at room temperature. Then, the cells were centrifuged, the supernatant was discarded, and the cells were washed twice with PBS $(350 \mu \mathrm{L})$. The samples were mixed and placed in a 96-well plate $(100 \mu \mathrm{L} /$ well $)$ with three replicate wells per concentration, and then the OD600 value was measured with an iMark microplate reader.

\section{Shigella Transcriptome Sequencing Analysis}

Total RNA was extracted from the tissue using TRIzol ${ }^{\circledR}$ reagent according to the manufacturer's instructions (Invitrogen), and genomic DNA was removed using DNase I (TaKara). The RNA-seq transcriptome library was prepared from $2 \mu \mathrm{g}$ of total RNA using the TruSeq ${ }^{\mathrm{TM}}$ RNA sample preparation kit from Illumina (San Diego, CA). The processing of original images to sequences, base calling, and quality value calculations were performed using the Illumina GA Pipeline (version 1.6), in which 150 bp paired-end reads were obtained. A Perl program was written to select clean reads by removing low-quality sequences, reads with more than $5 \% \mathrm{~N}$ bases (unknown bases) and reads containing adaptor sequences.

The data generated from the Illumina platform were used for the bioinformatics analysis. All analyses were performed using the I-Sanger Cloud Platform (www.i-san ger.com) from Shanghai Majorbio. After correction for multiple testing, we chose pathways with a P-value $\leq$ 0.05 that were significantly enriched in DEGs.

\section{Statistical Analysis}

Statistical analyses were performed using GraphPad Prism (GraphPad Software). Data are reported as the means \pm SD. Comparisons between two groups were assessed using Student's $t$-test or the Mann-Whitney test, depending on whether the data were normally distributed. Statistical significance between multiple groups was tested using one-way multiple analysis of variance (ANOVA) or the Kruskal-Wallis test followed by Tukey's test. The level of statistical significance was set to $p<0.05$.

\section{Results}

\section{The Antibacterial Activity of Au NCs Toward Strains Sf30I, R2448, and RII-I}

We synthesized $\mathrm{Au}$ NCs with MHA ligands with a diameter of $9 \mathrm{~nm}$ using the $\mathrm{NaBH}_{4}$ reduction method ${ }^{34,36}$ (Au NC particle size of $2 \mathrm{~nm}$, with good dispersibility) to evaluate the antibacterial activity of Au NCs. Different absorption peaks were observed at 670 and $760 \mathrm{~nm}^{36,40}$ We incubated the standard strain Sf301 and the clinically resistant bacterial strains R2448 and RII-1 with 0, 50, or $100 \mu \mathrm{M}$ Au NCs and used the colony-forming unit (CFU) method to 
quantitatively analyse the viability of Shigella. The growth of Shigella in the presence of different concentrations of $\mathrm{Au}$ NCs is shown in Figure 1 (the standard strain Sf301 was analysed after $1 \mathrm{~h}$ and $2 \mathrm{~h}$, clinically resistant bacteria R2448 and RII-1 were analysed after $2 \mathrm{~h}$ ). The plate counts of the three strains all showed that Au NCs almost completely inhibited the growth of Shigella (Figure 1), especially the clinically resistant bacteria R2448 (Figure 1B).

\section{The Antibacterial Activity of Au NCs}

The antibacterial effect of Au NCs on Shigella is reflected in the morphology of bacteria, as shown in Figure 2. Compared with the control group, the Shigella membrane was dissolved and the bacterial structure was disintegrated in the $\mathrm{Au}$ NC group. The internalization of Au NCs is the basis for killing Shigella, and the SEM image also indicates the inevitable effect of $\mathrm{Au}$ NCs on the cell membrane.

\section{Au NCs Alleviate the Clinical Symptoms of S. flexneri Enteritis}

We treated mice with $\mathrm{Au}$ NCs for 14 days and induced murine Shigella infectious colitis by administrating Shigella to clarify the role of Au NCs in Shigella infectious colitis (Figure 3A). The most important consideration for the development of new drugs is that their benefits to the human body outweigh the harms. We have concluded that $\mathrm{Au}$ NCs do not exert toxic side effects on human cells. ${ }^{36}$ In this experiment, we administered $100 \mu \mathrm{M} \mathrm{Au}$ NCs to healthy mice by gavage, observed the weight change in the mice, and obtained a 14-day mouse body weight curve (Figure 3B). No

A
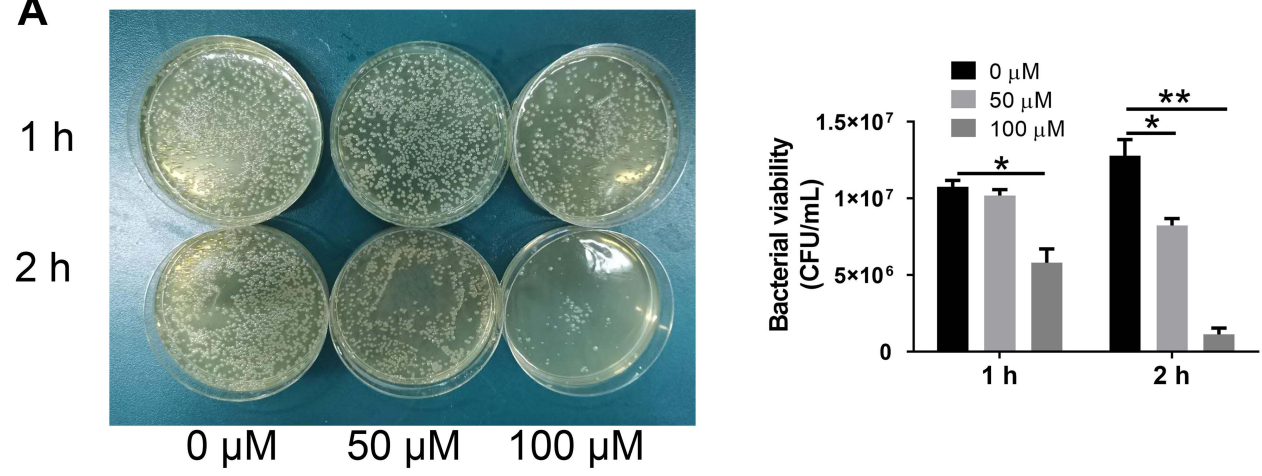

B
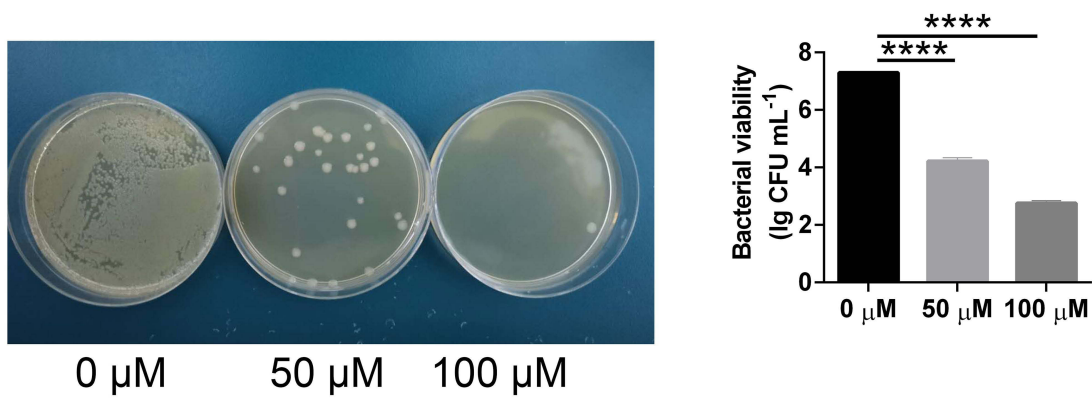

C
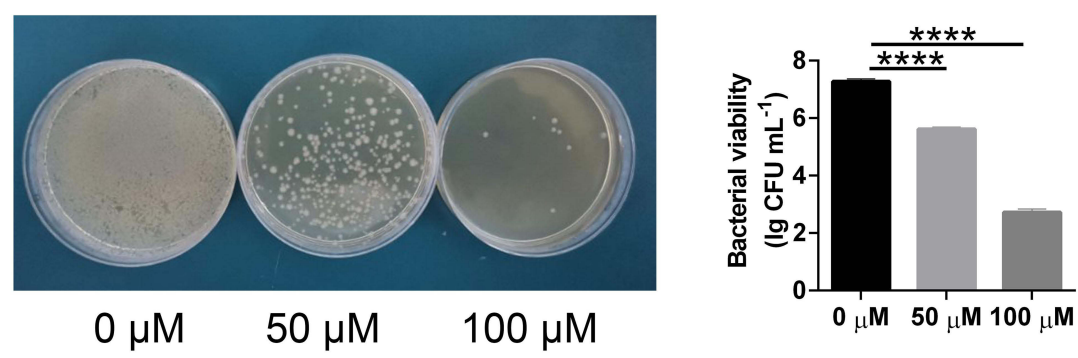

Figure I Antibacterial effect of Au NCs on the standard strain Sf30I (A), clinically resistant Shigella R2448 (B), and clinically resistant Shigella RII-I (C). Data are presented as the means $\pm \operatorname{SEM}(n=6)$. Statistical significance was determined with one-way ANOVA followed by Tukey's test, and $p$-values were calculated as follows: $* p<0.05$, $* * p<0.0$ I, $* * * * p<0.0001$. 


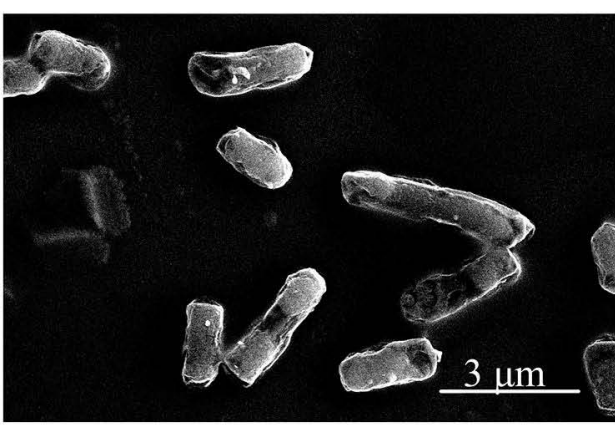

SF

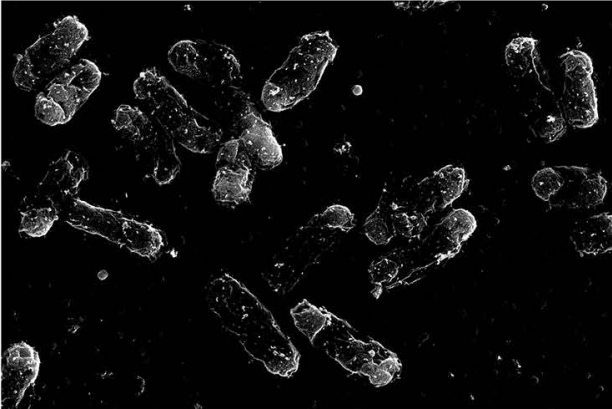

$\mathrm{SF}+\mathrm{AuNCs}$

Figure 2 Au NCs exert a strong killing effect on Shigella. Shigella were incubated with $100 \mu$ M Au NCs for 2 h. SEM images of Shigella before and after treatment with Au NCs and changes in the cell structure of Shigella. The scale bar represents $3 \mu \mathrm{m}$.
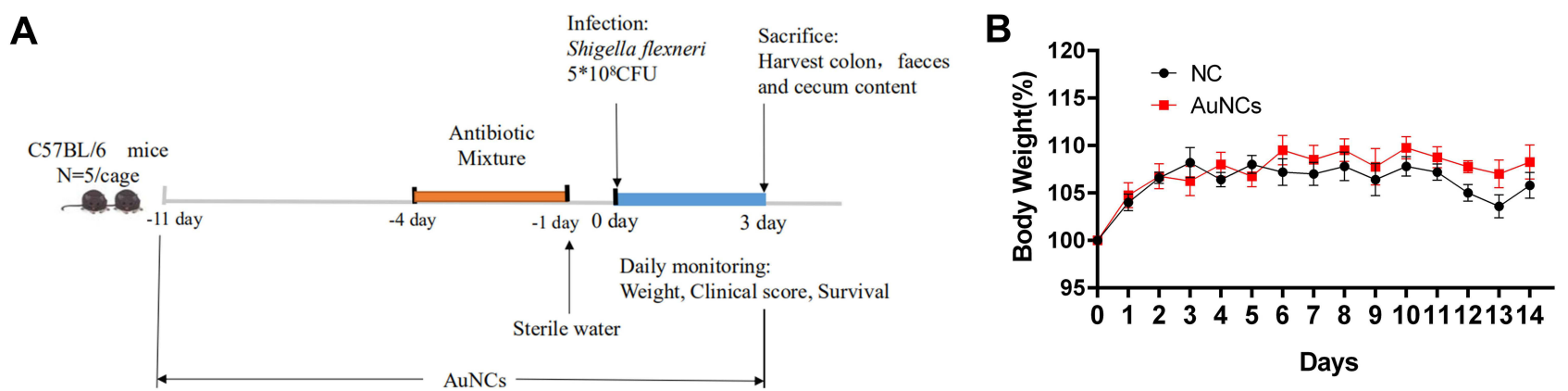

C

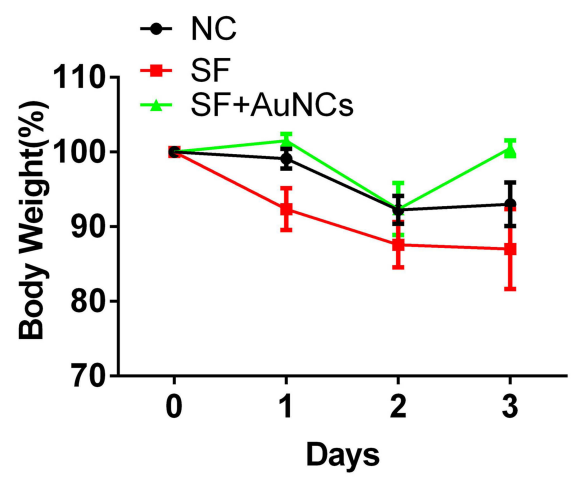

E

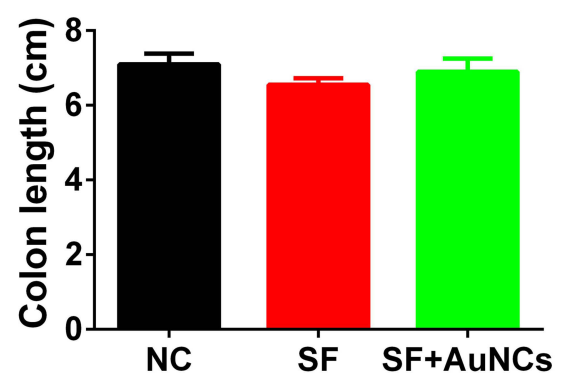

D

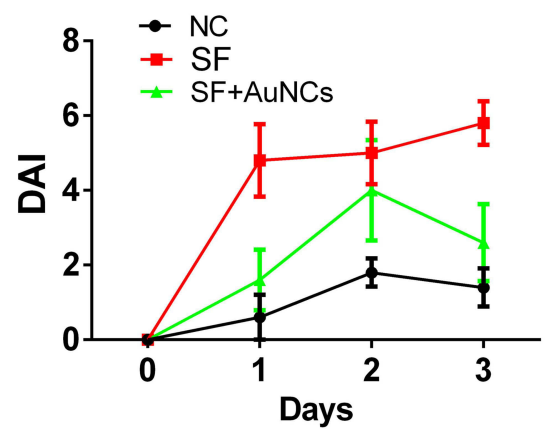

F

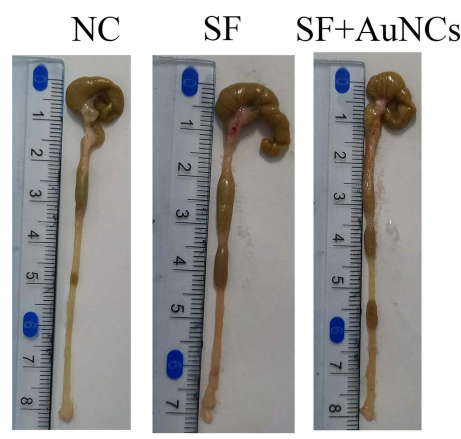

Figure 3 Au NCs alleviate the clinical symptoms of S. flexneri enteritis. (A) Mouse model of Shigella infection, (B) body weight changes of the NC group and the SF-Au NCs, (C) changes in body weight of mice from day 0 (the day of infection), (D) DAl scores of mice from day 0 (the day of infection), (E) statistical chart of mouse colon length, and (F) colon appearance of the NC group, the SF group and the SF-Au NCs group. Data are presented as the means \pm SEM ( $n=10$ ). Statistical significance was determined with one-way ANOVA followed by Tukey's test. 
significant difference in body weight was observed between $\mathrm{Au}$ NC-treated mice and control mice, which further proved the biocompatibility of $\mathrm{Au} \mathrm{NCs}$.

The body weight of $S$. flexneri-infected mice decreased substantially after Shigella infection. In the SF+Au NC group, weight loss did not occur immediately after Shigella infection, and weight recovered significantly on day 2. During the entire post infection period, the body weight of $\mathrm{Au} \mathrm{NC}$-treated mice was always greater than that of untreated mice, indicating a beneficial effect of $\mathrm{Au}$ NCs on Shigella enteritis (Figure 3C).

Due to Shigella infection, S. flexneri-infected mice presented clinical symptoms such as weight loss, diarrhoea and bloody stools, and the clinical disease activity index (DAI) increased. Au NC-treated mice showed a relatively smaller decrease in body weight and lower clinical scores than the SF group (Figure 3D). In conclusion, Au NCs improved the clinical symptoms of Shigella enteritis in mice.

Gross observation of the colon segment showed that $\mathrm{Au}$ NCs relieved Shigella enteritis, and the length of the colon in the $\mathrm{SF}+\mathrm{Au} \mathrm{NC}$ group was slightly increased compared with that in the SF group, which was not significantly different from that of the $\mathrm{NC}$ group (Figures 3E and F).

\section{Au NCs Relieve Intestinal Damage Caused by Shigella Infection}

Shigella-infected mice showed signs of colitis, such as weight loss, loose stools, colonic mucosa erosion, and crypt structure destruction. The colon glands of the mice treated with Au NCs were arranged neatly, the crypt structure was slightly damaged, and inflammatory cells were locally infiltrated, similar to the colon of the control group (Figure 4A). The histological damage score of the colon also showed that $\mathrm{Au}$ NCs significantly reduced colitis caused by Shigella infection (Figure 4B).

When the mice showed symptoms of infection, the faeces were collected to detect the LCN2 content. The results are shown in Figure 4C. Compared with mice in the SF group, the faecal LCN2 content of mice in the SF $+\mathrm{Au} \mathrm{NC}$ group was significantly decreased, indicating that colitis was effectively relieved.

\section{Au NC Treatment Reduces the Shigella Load in Faeces}

$\mathrm{Au}$ NCs showed strong antibacterial effects in vitro and relieved Shigella infection. Therefore, we further investigated whether they exerted an antibacterial effect on
A

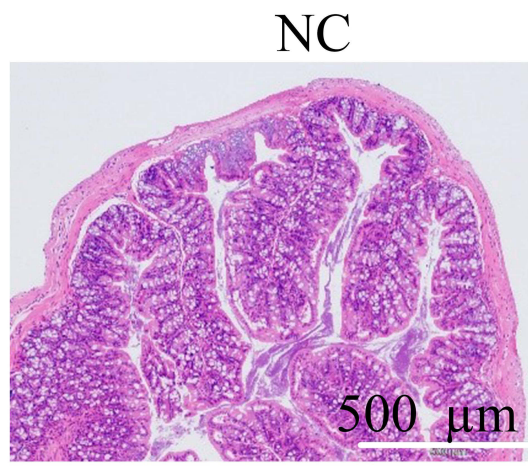

B

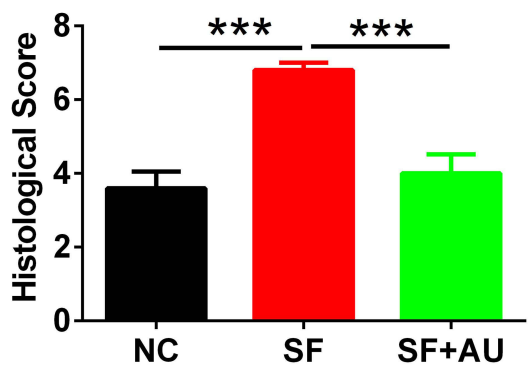

SF

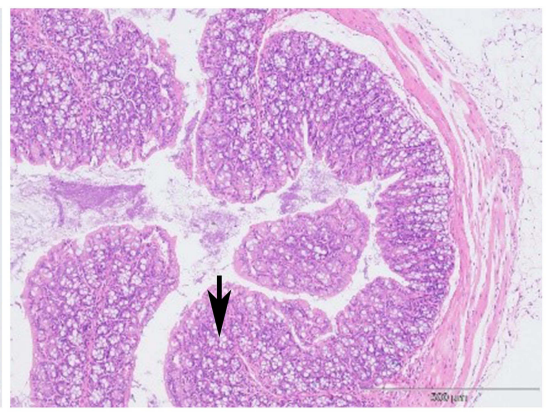

C

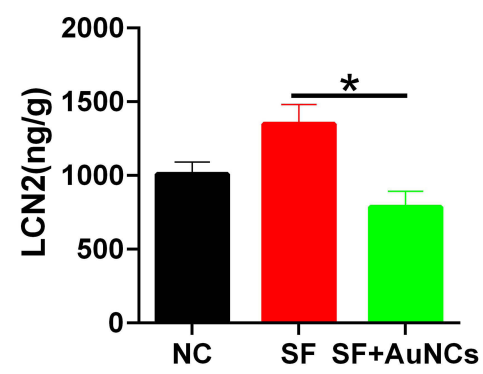

$\mathrm{SF}+\mathrm{AuNCs}$

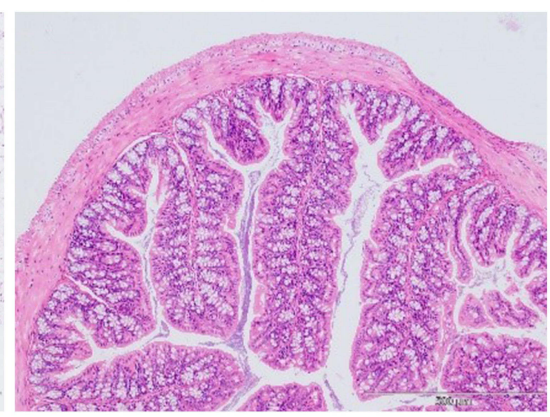

Figure 4 The effect of Au NCs on colon tissue damage and the faecal LCN2 content in mice infected with Shigella. (A) H\&E-stained pathological sections of the colon tissue $(100 \times)$, (B) histological score, and (C) faecal LCN2 content. Data are presented as the means \pm SEM $(n=10)$. Statistical significance was determined with one-way ANOVA followed by Tukey's test, and $p$-values are as follows: $* p<0.05$ and $* * * p<0.00 \mathrm{I}$. 
Shigella in vivo. The results of the bacterial load analysis in mice showed that Au NCs significantly reduced the CFU of Shigella in faeces compared with the SF group (Figure 5), indicating that Au NCs inhibited Shigella and alleviated Shigella enteritis.

\section{Au NCs Reduce Shigella Biofilm Activity}

A biofilm is a common infection mode of Shigella, a foodborne bacterium, and it is also a protective umbrella for Shigella. Biofilms have natural resistance to antibiotics, which substantially reduces their sensitivity to antibiotics. ${ }^{41}$ We tried to study whether the biofilm formed by Shigella was also an impenetrable wall for Au NCs. As shown in Figure 6, $100 \mu \mathrm{M} \mathrm{Au}$ NCs reduced the biofilm activity of Shigella to approximately $60 \%$, suggesting that the application of $\mathrm{Au}$ NCs will not encounter resistance due to biofilm formation.

\section{Au NCs Affect Metabolism-Related Genes and Induce ROS Release in Shigella} After being internalized by bacteria, Au NCs affect the metabolism of bacteria and induce excess ROS release. As shown in Figure 7A, Au NCs increased the level of ROS metabolized by Shigella by approximately 5 times. This effect is the main antibacterial pathway of Au NCs, as excessive ROS directly kill bacteria. ${ }^{34}$ After testing the expression of metabolism-related genes, as shown in Figure 7B, we found that $\mathrm{Au}$ NCs downregulated antioxidant genes such as ompA, gshA, bssR, elaB, alkB, grxA, nth, relB, and nuoF and upregulated oxidative stress genes such as copA, htrA, soxS, and hha (Table S4), which may have led to a substantial increase in ROS production. We decomposed the predicted metagenomic information into KEGG (Kyoto Encyclopedia of Genes and Genomes) and GO (Gene Ontology) pathways. Treatment with Au NCs mainly caused changes in the tricarboxylic acid cycle and cell metabolism (including pyrimidine metabolism, purine

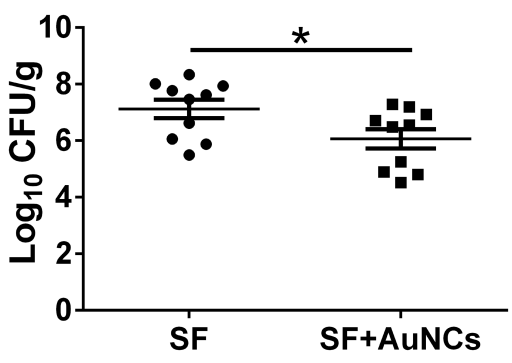

Figure 5 The effect of Au NCs on the Shigella load (faeces). Data are presented as the means $\pm \operatorname{SEM}(n=10)$. Statistical significance was determined with one-way ANOVA followed by Tukey's test, and $p$-values are as follows: ${ }^{*} p<0.05$.

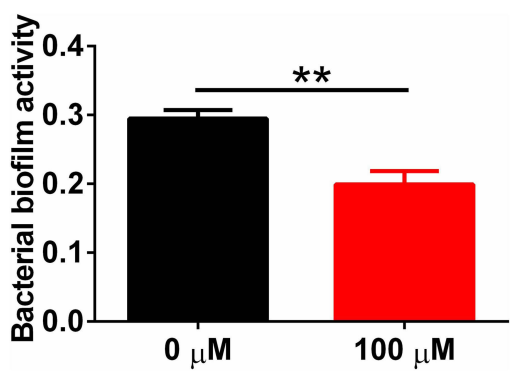

Figure 6 The effect of Au NCs on Shigella biofilm activity. Data are presented as the means \pm SEM $(n=6)$. Statistical significance was determined with one-way ANOVA followed by Tukey's test, and $p$-values are as follows: $*^{*} p<0.01$.

metabolism, and nucleotide metabolism), cell membrane transport, signal transduction and other pathways and changes in gene abundance (Figures $7 \mathrm{C}$ and $\mathrm{D}$, Supplementary Figure 1), which may be involved in the release of ROS and cell membrane integrity.

\section{Discussion}

The global financial burden caused by Shigella infection cannot be ignored, and its multidrug resistance has hindered effective antibiotic treatment. The remarkable antibacterial properties and excellent therapeutic effects of $\mathrm{Au}$ NCs reported in this study have once again ushered in the dawn of the treatment of Shigella infectious diseases. We used the NaBH4 reduction method to synthesize Au NCs. The process is energy saving, and the synthesis is fast, making it highly stable The synthesized Au NCs have an ultrasmall size and large specific surface area, rendering the surface highly active. ${ }^{34,37}$

We incubated Au NCs with Sf301 and the clinically resistant strains R2448 and RII-1 to develop a drug with antibacterial activity that does not produce drug resistance. These two strains of resistant bacteria are resistant to almost all antibiotics and are representative strains. Remarkably, $100 \mu \mathrm{M}$ Au NCs exerted obvious antibacterial effects on these three strains and basically inhibited the growth of $100 \%$ of the colonies, indicating that $\mathrm{Au} \mathrm{NCs}$ may not be affected by the resistance mechanism of Shigella. SEM was used to determine the structure of Shigella in order to prove the effectiveness of Au NCs, and studies have shown that after nanoparticles are in contact with microorganisms, nanoparticles first attack the outermost structure of the microbe cells. ${ }^{42}$ Similar to the role of $\mathrm{Au}$ NCs in Clostridium difficile, the SEM images we obtained and the detection of the changes in the abundance of membrane-related genes proved that $\mathrm{Au}$ NCs dissolved the Shigella membrane. This effect may be 
A

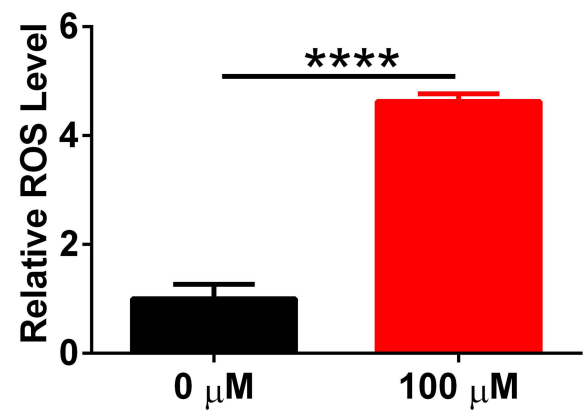

C

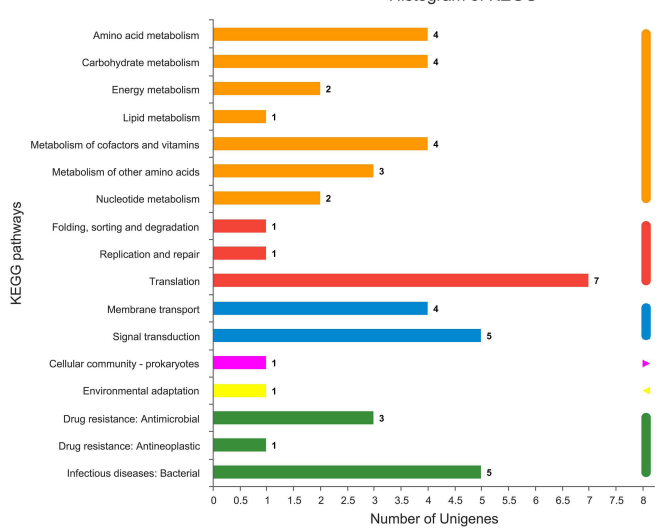

NC_vs_Au.volcano
B

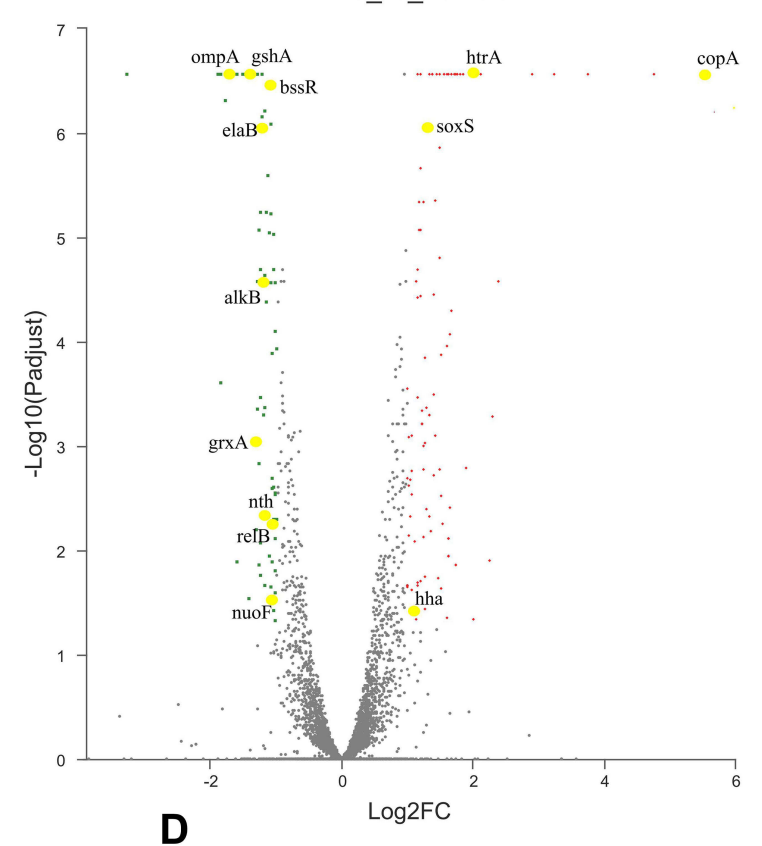

nosig up

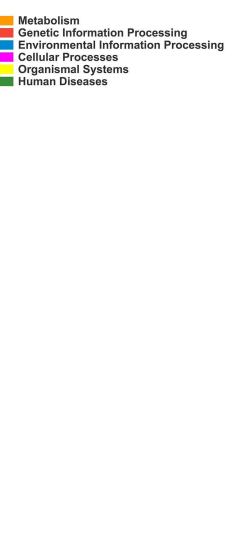

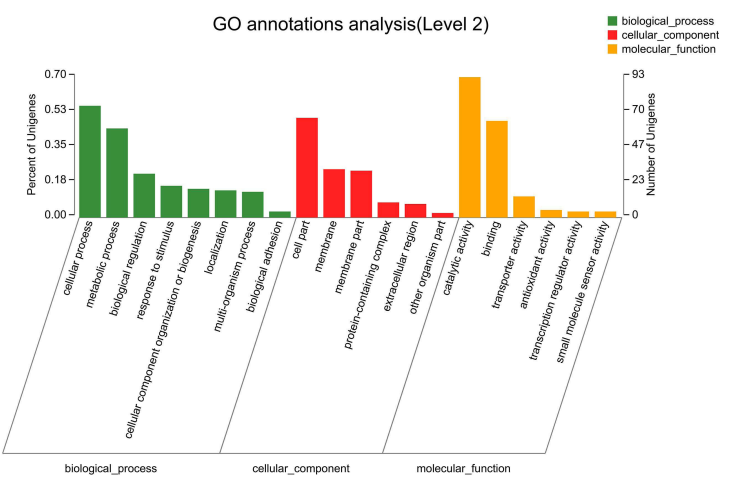

Figure $7 \mathrm{Au}$ NCs affect the cellular functions of Shigella and kill the cells. Au NCs induced an increase in intracellular ROS levels (A). Volcano map of changes in ROS metabolism-related gene expression after Au NC treatment. Red indicates that the gene is upregulated, and green indicates that the gene is downregulated (B). Diagram of the KEGG analysis of genes related to cell metabolism, substrate transport, membrane integrity and cell transcriptome processes after Au NC treatment (C). After the Au NC treatment, a GO analysis of genes related to cell metabolism, substrate transport, membrane integrity and cell transcriptome processes was performed (D). Data are presented as the means $\pm \operatorname{SEM}(n=6)$. Student's $t$-test was used for statistical analyses, and $p$-values are as follows: $* * * * p<0.000 \mathrm{I}$.

mediated by the free radicals generated in response to $\mathrm{Au}$ NCs, which is a characteristic of metals. ${ }^{36,43}$ Most interestingly, scientists have shown that the stronger effect of $\mathrm{Au}$ NCs on gram-negative bacteria is because gramnegative bacteria lack the hard cell wall network structure of the peptidoglycan layer of gram-positive bacteria, ${ }^{44}$ making them more susceptible to mechanical damage by $\mathrm{Au} \mathrm{NCs}$ that further interact with the membrane. Shigella is exactly this type of bacteria.

We established Shigella-induced colitis mouse models and compared the therapeutic effects of $\mathrm{Au}$ NCs between the SF group and SF-Au NC group to prove the feasibility of $\mathrm{Au}$ NCs. Judging from the clinical signs of the mice, including the mouse body weight, DAI, and colon length, the mice almost recovered their health after treatment with Au NCs. We investigated the curative effect more deeply. The tissue damage score basically returned to normal. The intestinal mucosa and epithelial cells damaged by Shigella gradually recovered in mice treated with Au NCs. The increase in goblet cells was evidence of recovery (the mucus secreted by goblet cells provides the protective layer of the intestinal barrier). ${ }^{45}$ The bacterial load in mouse faeces also decreased significantly after treatment with $\mathrm{Au}$ NCs. In individuals with colitis, the content of LCN2 is usually increased, and LCN2 exerts an antiinflammatory effect. ${ }^{46} \mathrm{Au}$ NCs reduced the faecal LCN2 
content, which further proves that $\mathrm{Au}$ NCs represent a treatment for Shigella-induced colitis in mice.

We then conducted an in-depth study of the antibacterial mechanism of Au NCs. Surprisingly, Au NCs affected Shigella biofilms and reduced their activity. Biofilm refers to a special bacterial structure attached to the surface of an object and wrapped by bacterial extracellular macromolecules. Biofilms protect invading bacteria from being discovered by the immune system. In addition, biofilms cause chronic infections. Moreover, ordinary antibiotics do not penetrate the biofilm to attack Shigella. The existence of biofilms makes bacteria 1000 times more resistant. ${ }^{47-49}$ Biofilms are an obstacle that must be overcome in the treatment of Shigella infection. Studies have shown that nanoparticles prevent the production of biofilms, ${ }^{50}$ and thus we explored whether Au NCs affected Shigella biofilms. The results proved the unexpected antibacterial power of $\mathrm{Au} \mathrm{NCs}$ against Shigella. $\mathrm{Au} \mathrm{NCs}$ are also expected to pass through the biofilms of Pseudomonas aeruginosa and Escherichia coli to kill pathogens, ${ }^{51,52}$ potentially because tiny particles destroy the stability of the biofilm through some mechanisms. ${ }^{53}$ We further explored the mechanism by focusing on ROS, which are essential for antibacterial activity. Many effective antibiotics achieve antibacterial effects by increasing the ROS level in bacteria. ${ }^{54}$ ROS include superoxide $\left(\mathrm{O}^{2-}\right)$, hydrogen peroxide $\left(\mathrm{H}_{2} \mathrm{O}_{2}\right)$, hydroxyl radicals $\left(\mathrm{OH}^{-}\right)$, singlet oxygen, and lipid hydroperoxides. Among them, $\mathrm{H}_{2} \mathrm{O}_{2}$ plays an important antibacterial role; ${ }^{55}$ therefore, we used DCFH reagent to detect the $\mathrm{ROS} \mathrm{H}_{2} \mathrm{O}_{2}$. The mechanism of antibiotics is to interfere with the tricarboxylic acid cycle through the electron transport chain, thereby affecting cellular metabolism and inducing ROS generation. ${ }^{56}$ Our detection of the metabolic gene abundance also proves this pathway. ROS are a natural by-product of energy produced by mitochondria that play an important role in cellular signal transduction and homeostasis. However, during periods of environmental stress, ROS levels increase substantially, ${ }^{57}$ especially $\mathrm{H}_{2} \mathrm{O}_{2}$, and bacteria must use $\mathrm{H}_{2} \mathrm{O}_{2}$ to balance stimulation. ${ }^{58}$ However, once ROS are released in large quantities and exceed a certain limit, they produce oxidative stress, killing bacteria by interfering with proteins, DNA, lipids and other macromolecular substances. ${ }^{59,60}$ In addition, ROS can control the bacterial load and destroy biofilms, and studies have proven that nanoparticles have the characteristics of antimicrobial drug resistance, which mainly depends on ROS. ${ }^{61}$ The antimicrobial drug resistance of Au NCs also mainly depends on
ROS, and studies have shown that ROS even become an antibacterial substance alone. ${ }^{62}$ Moreover, the potential healing effect of $\mathrm{Au} \mathrm{NCs}$ on a variety of cancer cells, such as prostate cancer, ${ }^{63,64}$ is mostly based on ROS. We quantitatively detected ROS-related genes and observed decreased expression of ompA, gshA, bssR, elaB, alkB, grxA, nth, relB, nuoF and other antioxidant genes, ${ }^{65-72}$ whereas the expression of $\operatorname{copA}, \mathrm{htrA}$, soxS, hha and other oxidative stress genes $^{71,73-77}$ was upregulated. These changes also contribute to the mechanism by which $\mathrm{Au}$ NCs induce ROS production.

Overall, in the era of antibiotic abuse and universal drug resistance of pathogenic microorganisms, Au NCs will have a place in the treatment of microbial infections in the near future due to their antibacterial activity and antidrug resistance, as well as their biological safety. Au $\mathrm{NC}$ treatment of Shigella infectious diseases is expected in the near future. However, further experimental studies are needed to determine whether $\mathrm{Au}$ NCs are resistant to other probiotics in vivo and the dosage of Au NCs.

\section{Abbreviations}

NCs, nanoclusters; SEM, scanning electron microscope; DAI, disease activity index; LCN2, lipocalin-2; ROS, reactive oxygen species; S. flexneri, Shigella flexneri; SPR, surface plasmon resonance; MHA, mercaptohexanoic acid; TEM, transmission electron microscopy; DLS, dynamic light scattering; UV-Vis, ultraviolet-visible; TSB, Trypticase (Tryptic) Soy Broth; PBS, phosphate buffered solution; NC, normal control group; SF, S. flexneri infection group; SF-Au $\mathrm{NCs}, \mathrm{Au}$ NCs treatment group; H\&E, hematoxylin-eosin; EP, eppendorf; CFU, colony-forming unit; KEGG, Kyoto Encyclopedia of Genes and Genomes; GO, Gene Ontology.

\section{Acknowledgments}

Xiaoxiao $\mathrm{Wu}$, Yongyan Chen and Yangheng Zhang shared co-first authorship. This research was supported by the National Natural Science Foundation of China (81871734, 81471994), the Xuzhou Science and Technology planning Project (KC20116), Natural Science Research Project of Higher Education Institutions in Jiangsu Province (20KJB310013) and Xuzhou Medical University Excellent Talent Introduction Project (D2019030).

\section{Disclosure}

The authors declare no conflict of interest. 


\section{References}

1. Trofa AF, Ueno-Olsen H, Oiwa R, et al. Dr. Kiyoshi Shiga: discoverer of the dysentery bacillus. Clin Infect Dis. 1999;29 (5):1303-1306. doi:10.1086/313437

2. Kotloff KL, Winickoff JP, Ivanoff B, et al. Global burden of Shigella infections: implications for vaccine development and implementation of control strategies. Bull World Health Organ. 1999;77(8):651-666.

3. Bardhan P, Faruque AS, Naheed A, et al. Decrease in shigellosis-related deaths without Shigella spp.-specific interventions, Asia. Emerg Infect Dis. 2010;16(11):1718-1723. doi:10.3201/ eid1611.090934

4. Tickell KD, Brander RL, Atlas HE, et al. Identification and management of Shigella infection in children with diarrhoea: a systematic review and meta-analysis. Lancet Glob Health. 2017;5(12):e1235e1248. doi:10.1016/S2214-109X(17)30392-3

5. Troeger C, Forouzanfar M, Rao PC; GBD Diarrhoeal Diseases Collaborators. Estimates of global, regional, and national morbidity, mortality, and aetiologies of diarrhoeal diseases: a systematic analysis for the Global Burden of Disease Study 2015. Lancet Infect Dis. 2017;17(9):909-948. doi:10.1016/S1473-3099(17)30276-1

6. Kotloff KL. The burden and etiology of diarrheal illness in developing countries. Pediatr Clin North Am. 2017;64(4):799-814. doi:10.1016/j.pcl.2017.03.006

7. Nisa I, Qasim M, Yasin N, et al. Shigella flexneri: an emerging pathogen. Folia Microbiol. 2020;65(2):275-291. doi:10.1007/ s12223-020-00773-w

8. Niyogi SK. Shigellosis. J Microbiol. 2005;43(2):133-143.

9. Singh A, Barnard TG. Adaptations in the physiological heterogeneity and viability of Shigella dysenteriae, Shigella flexneri and Salmonella typhimurium, after exposure to simulated gastric acid fluid. Microb Pathog. 2017;113:378-384. doi:10.1016/j.micpath.2017.11.014

10. Mathan MM, Mathan VI. Ultrastructural pathology of the rectal mucosa in Shigella dysentery. Am J Pathol. 1986;123(1):25-38.

11. Ashida H, Ogawa M, Mimuro H, et al. Shigella infection of intestinal epithelium and circumvention of the host innate defense system. Curr Top Microbiol Immunol. 2009;337:231-255. doi:10.1007/978-3-64201846-6 8

12. Liu G, Pilla G, Tang CM. Shigella host: pathogen interactions: keeping bacteria in the loop. Cell Microbiol. 2019;21(11):e13062. doi: $10.1111 / \mathrm{cmi} .13062$

13. Prantner D, Nagarajan UM. Role for the chlamydial type III secretion apparatus in host cytokine expression. Infect Immun. 2009;77 (1):76-84. doi:10.1128/IAI.00963-08

14. Harada A, Sekido N, Akahoshi T, et al. Essential involvement of interleukin-8 (IL-8) in acute inflammation. J Leukoc Biol. 1994;56 (5):559-564. doi:10.1002/jlb.56.5.559

15. Sakaguchi T, Köhler H, Gu X, et al. Shigella flexneri regulates tight junction-associated proteins in human intestinal epithelial cells. Cell Microbiol. 2002;4(6):367-381. doi:10.1046/j.1462-5822.2002.00197.x

16. Schuster HJ, Gompelman M, Ang W, et al. An adult case with shigellosis-associated encephalopathy. BMJ Case Rep. 2018;2018: bcr2017222372. doi:10.1136/bcr-2017-222372

17. Kang L, Millett PJ, Mezera K, et al. Chronic plasma cell osteomyelitis of the humerus associated with Shigella and Flavobacterium. J Shoulder Elbow Surg. 2001;10(3):292-294. doi:10.1067/mse.2001.113084

18. Miron D, Sochotnick I, Yardeni D, et al. Surgical complications of shigellosis in children. Pediatr Infect Dis J. 2000;19(9):898-900. doi:10.1097/00006454-200009000-00022

19. World Health Organization. The selection and use of essential medicines. 2017. Available from: http://www.who.int/medicines/pub lications/essentialmedicines/en/. Accessed August 3, 2017.

20. Baker S, The HC. Recent insights into Shigella: a major contributor to the global diarrhoeal disease burden. Curr Opin Infect Dis. 2018;31(5):449-454. doi:10.1097/QCO.0000000000000475
21. Puzari M, Sharma M, Chetia P. Emergence of antibiotic resistant Shigella species: a matter of concern. J Infect Public Health. 2018;11(4):451-454. doi:10.1016/j.jiph.2017.09.025

22. Song Y, Sun M, Feng L, et al. Antibiofilm activity of Lactobacillus plantarum 12 exopolysaccharides against Shigella flexneri. Appl Environ Microbiol. 2020;86(15):e00694-20. doi:10.1128/ AEM.00694-20

23. Raqib R, Sarker P, Mily A, et al. Efficacy of sodium butyrate adjunct therapy in shigellosis: a randomized, double-blind, placebo-controlled clinical trial. BMC Infect Dis. 2012;12:111. doi:10.1186/1471-2334-12-111

24. Hsu BB, Way JC, Silver PA. Stable neutralization of a virulence factor in bacteria using temperate phage in the mammalian gut. mSystems. 2020;5(1):e00013-e00020. doi:10.1128/mSystems.00013-20

25. Barel LA, Mulard LA. Classical and novel strategies to develop a Shigella glycoconjugate vaccine: from concept to efficacy in human. Hum Vaccin Immunother. 2019;15(6):1338-1356. doi:10.1080/21645515.2019.1606972

26. Kong FY, Zhang JW, Li RF, et al. Unique roles of gold nanoparticles in drug delivery, targeting and imaging applications. Molecules. 2017;22(9):1445. doi:10.3390/molecules22091445

27. Iglesias E. Gold nanoparticles as colorimetric sensors for the detection of DNA bases and related compounds. Molecules. 2020;25 (12):2890. doi:10.3390/molecules 25122890

28. Yahaya ML, Zakaria ND, Noordin R, et al. Development of rapid gold nanoparticles based lateral flow assays for simultaneous detection of Shigella and Salmonella genera. Biotechnol Appl Biochem. 2020. doi:10.1002/bab.2029

29. Elahi N, Kamali M, Baghersad MH, et al. A fluorescence nano-biosensors immobilization on iron (MNPs) and gold (AuNPs) nanoparticles for detection of Shigella spp. Mater Sci Eng C Mater Biol Appl. 2019;105:110113. doi:10.1016/j.msec.2019.110113

30. Wang C, Wang Y, Zhang L, et al. Pretreated macrophage-membranecoated gold nanocages for precise drug delivery for treatment of bacterial infections. Adv Mater. 2018;30(46):e1804023. doi:10.1002/ adma.201804023

31. Wang P, Zhang L, Zheng W, et al. Thermo-triggered release of CRISPR-Cas9 system by lipid-encapsulated gold nanoparticles for tumor therapy. Angew Chem Int Ed. 2018;57(6):1491-1496. doi:10.1002/anie.201708689

32. Hussain S, Joo J, Kang J, et al. Antibiotic-loaded nanoparticles targeted to the site of infection enhance antibacterial efficacy. Nat Biomed Eng. 2018;2(2):95-103. doi:10.1038/s41551-0170187-5

33. Yougbare $\mathrm{S}$, Chang TK, Tan SH, et al. Antimicrobial gold nanoclusters: recent developments and future perspectives. Int J Mol Sci. 2019;20(12):2924. doi:10.3390/ijms20122924

34. Zheng K, Setyawati MI, Leong DT, et al. Antimicrobial gold nanoclusters. ACS Nano. 2017;11(7):6904-6910. doi:10.1021/ acsnano.7b02035

35. Hammer B, Norskov J. Why gold is the noblest of all the metals. Nature. 1995;376:238. doi:10.1038/376238a0

36. Yang H, Cai R, Zhang Y, et al. Gold nanoclusters as an antibacterial alternative against clostridium difficile. Int $J$ Nanomedicine. 2020;15:6401-6408. doi:10.2147/IJN.S268758

37. Vijayan R, Joseph S, Mathew B. Eco-friendly synthesis of silver and gold nanoparticles with enhanced antimicrobial, antioxidant, and catalytic activities. IET Nanobiotechnol. 2018;12(6):850-856. doi:10.1049/iet-nbt.2017.0311

38. Xu D, Liao C, Xiao J, et al. Human enteric defensin 5 promotes Shigella infection of macrophages. Infect Immun. 2019;88(1): e00769-19. doi:10.1128/IAI.00769-19

39. National Research Council (US) Committee for the Update of the Guide for the Care and Use of Laboratory Animals. Guide for the Care and Use of Laboratory Animals. 8th ed. Washington (DC): National Academies Press (US); 2011. 
40. Yuan X, Zhang B, Luo Z, et al. Balancing the rate of cluster growth and etching for gram-scale synthesis of thiolate-protected $\mathrm{Au}(25)$ nanoclusters with atomic precision. Angew Chem Int Ed. 2014;53 (18):4623-4627. doi:10.1002/anie.201311177

41. Hadadi-Fishani M, Khaledi A, Fatemi-Nasab ZS. Correlation between biofilm formation and antibiotic resistance in Pseudomonas aeruginosa: a meta-analysis. Infez Med. 2020;28(1):47-54.

42. Reddy GB, Madhusudhan A, Ramakrishna D, et al. Green chemistry approach for the synthesis of gold nanoparticles with gum kondagogu: characterization, catalytic and antibacterial activity. J Nanostructure Chem. 2015;5(2):185-193. doi:10.1007/s40097-015-0149-y

43. Kim JS, Kuk E, Yu KN, et al. Antimicrobial effects of silver nanoparticles - ScienceDirect. Nanomedicine: NBM. 2007;3(1):95-101. doi:10.1016/j.nano.2006.12.001

44. Elemike EE, Onwudiwe DC, Fayemi OE, et al. Biosynthesis, electrochemical, antimicrobial and antioxidant studies of silver nanoparticles mediated by talinum triangulare aqueous leaf extract. $J$ Cluster Sci. 2017;28(1):1-22. doi:10.1007/s10876-016-1087-7

45. Cornick S, Kumar M, Moreau F, et al. VAMP8-mediated MUC2 mucin exocytosis from colonic goblet cells maintains innate intestinal homeostasis. Nat Commun. 2019;10(1):4306. doi:10.1038/s41467019-11811-8

46. Moschen AR, Gerner RR, Wang J, et al. Lipocalin 2 protects from inflammation and tumorigenesis associated with gut microbiota alterations. Cell Host Microbe. 2016;19(4):455-469. doi:10.1016/j. chom.2016.03.007

47. Høiby N, Bjarnsholt T, Givskov M, et al. Antibiotic resistance of bacterial biofilms. Int $J$ Antimicrob Agents. 2010;35(4):322-332. doi:10.1016/j.ijantimicag.2009.12.011

48. Venkatesan N, Perumal G, Doble M. Bacterial resistance in biofilm-associated bacteria. Future Microbiol. 2015;10 (11):1743-1750. doi:10.2217/fmb.15.69

49. Roy R, Tiwari M, Donelli G, et al. Strategies for combating bacterial biofilms: a focus on anti-biofilm agents and their mechanisms of action. Virulence. 2018;9(1):522-554. doi:10.1080/21505594.20 17.1313372

50. Joshi AS, Singh P, Mijakovic I. Interactions of gold and silver nanoparticles with bacterial biofilms: molecular interactions behind inhibition and resistance. Int $J$ Mol Sci. 2020;21(20):7658. doi:10.3390/ijms21207658

51. Ali SG, Ansari MA, Alzohairy MA, et al. Biogenic gold nanoparticles as potent antibacterial and antibiofilm nano-antibiotics against pseudomonas aeruginosa. Antibiotics. 2020;9(3):100. doi:10.3390/ antibiotics 9030100

52. Li Y, Zhen J, Tian Q, et al. One step synthesis of positively charged gold nanoclusters as effective antimicrobial nanoagents against multidrug-resistant bacteria and biofilms. J Colloid Interface Sci. 2020;569:235-243. doi:10.1016/j.jcis.2020.02.084

53. Chaw C, Manimaran M, Tay FEH. Role of silver ions in destabilization of intermolecular adhesion forces measured by atomic force microscopy in Staphylococcus epidermidis biofilms. Antimicrob Agents Chemother. 2005;49:4853-4859. doi:10.1128/AAC.49.12.48 53-4859.2005

54. Van Acker H, Coenye T. The role of reactive oxygen species in antibiotic-mediated killing of bacteria. Trends Microbiol. 2017;25 (6):456-466. doi:10.1016/j.tim.2016.12.008

55. Lam PL, Wong RS, Lam KH. The role of reactive oxygen species in the biological activity of antimicrobial agents: an updated mini review. Chem Biol Interact. 2020;320:109023. doi:10.1016/j. cbi.2020.109023

56. Kohanski MA, Dwyer DJ, Hayete B, et al. A common mechanism of cellular death induced by bactericidal antibiotics. Cell. 2007;130 (5):797-810. doi:10.1016/j.cell.2007.06.049

57. Kausar S, Wang F, Cui $\mathrm{H}$. The role of mitochondria in reactive oxygen species generation and its implications for neurodegenerative diseases. Cells. 2018;7(12):274. doi:10.3390/cells7120274
58. Mailloux RJ. An update on mitochondrial reactive oxygen species production. Antioxidants. 2020;9(6):472. doi:10.3390/antiox9060472

59. Kim T, Zhang Q, Li J, et al. A gold/silver hybrid nanoparticle for treatment and photoacoustic imaging of bacterial infection. ACS Nano. 2018;12(6):5615-5625. doi:10.1021/acsnano.8b01362

60. Su LJ, Zhang JH, Gomez H, et al. Reactive oxygen species-induced lipid peroxidation in apoptosis, autophagy, and ferroptosis. Oxid Med Cell Longev. 2019;2019:5080843. doi:10.1155/2019/5080843

61. Pelgrift RY, Friedman AJ. Nanotechnology as a therapeutic tool to combat microbial resistance. Adv Drug Deliv Rev. 2013;65(1314):1803-1815. doi:10.1016/j.addr.2013.07.011

62. Dryden M. Reactive oxygen species: a novel antimicrobial. Int $J$ Antimicrob Agents. 2018;51(3):299-303. doi:10.1016/j. ijantimicag.2017.08.029

63. Lu L, Li K, Mao YH, et al. Gold-chrysophanol nanoparticles suppress human prostate cancer progression through inactivating AKT expression and inducing apoptosis and ROS generation in vitro and in vivo. Int J Oncol. 2017;51(4):1089-1103. doi:10.3892/ijo.2017.4095

64. de Sá Junior PL, Câmara DAD, Porcacchia AS, et al. The roles of ROS in cancer heterogeneity and therapy. Oxid Med Cell Longev. 2017;2017:2467940. doi:10.1155/2017/2467940

65. Shimada K, Nakamura M, Anai S, et al. A novel human AlkB homologue, ALKBH8, contributes to human bladder cancer progression. Cancer Res. 2009;69(7):3157-3164. doi:10.1158/00085472.CAN-08-3530

66. Harrison JJ, Tremaroli V, Stan MA, et al. Chromosomal antioxidant genes have metal ion-specific roles as determinants of bacterial metal tolerance. Environ Microbiol. 2009;11(10):2491-2509. doi:10.1111/ j.1462-2920.2009.01973.x

67. Walch M, Dotiwala F, Mulik S, et al. Cytotoxic cells kill intracellular bacteria through granulysin-mediated delivery of granzymes. Cell. 2015;161(5):1229. doi:10.1016/j.cell.2015.05.021

68. Liu TF, Vachharajani V, Millet P, et al. Sequential actions of SIRT1-RELB-SIRT3 coordinate nuclear-mitochondrial communication during immunometabolic adaptation to acute inflammation and sepsis. $J$ Biol Chem. 2015;290(1):396-408. doi:10.1074/jbc. M114.566349

69. Tang G, Xing S, Wang S, et al. Regulation of cysteine residues in LsrB proteins from Sinorhizobium meliloti under free-living and symbiotic oxidative stress. Environ Microbiol. 2017;19 (12):5130-5145. doi:10.1111/1462-2920.13992

70. Dai L, Xia J, Sahin O, et al. Identification of a nth-like gene encoding an endonuclease III in campylobacter jejuni. Front Microbiol. 2019;10:698. doi:10.3389/fmicb.2019.00698

71. Sulaiman JE, Lam H. Proteomic study of the survival and resuscitation mechanisms of filamentous persisters in an evolved Escherichia coli population from cyclic ampicillin treatment. mSystems. 2020;5 (4):e00462-20. doi:10.1128/mSystems.00462-20

72. Shivaprasad DP, Taneja NK, Lakra A, et al. In vitro and in situ abrogation of biofilm formation in $\mathrm{E}$. coli by vitamin $\mathrm{C}$ through ROS generation, disruption of quorum sensing and exopolysaccharide production. Food Chem. 2021;341(Pt1):128171. doi:10.1016/j. foodchem.2020.128171

73. Dong TG, Dong S, Catalano C, et al. Generation of reactive oxygen species by lethal attacks from competing microbes. Proc Natl Acad Sci U S A. 2015;112(7):2181-2186. doi:10.1073/pnas. 1425007112

74. Paul P, Verma S, Kumar Panda P, et al. Molecular insight to influential role of Hha-TomB toxin-antitoxin system for antibacterial activity of biogenic silver nanoparticles. Artif Cells Nanomed Biotechnol. 2018;46(sup3):S572-S584. doi:10.1080/21691401.2018.1503598

75. You H, Jin Y, Kang J, et al. Mitochondrial serine protease Omi/HtrA2 accentuates brain ischemia/reperfusion injury in rats and oxidative stress injury in vitro by modulating mitochondrial stress proteins $\mathrm{CHOP}$ and $\mathrm{ClpP}$ and physically interacting with mitochondrial fusion protein OPA1. Bioengineered. 2020;11(1):1058-1070. doi:10.1080/ 21655979.2020.1822105 
76. Tiku V, Kofoed EM, Yan D, et al. Outer membrane vesicles containing OmpA induce mitochondrial fragmentation to promote pathogenesis of Acinetobacter baumannii. Sci Rep. 2021;11(1):618. doi:10.1038/s41598-020-79966-9
77. Águila-clares B, Castiblanco LF, Quesada JM, et al. Transcriptional response of Erwinia amylovora to copper shock: in vivo role of the copA gene. Mol Plant Pathol. 2018;19(1):169-179. doi:10.1111/ mpp. 12510

\section{Publish your work in this journal}

The International Journal of Nanomedicine is an international, peerreviewed journal focusing on the application of nanotechnology in diagnostics, therapeutics, and drug delivery systems throughout the biomedical field. This journal is indexed on PubMed Central, MedLine, CAS, SciSearch ${ }^{\circledR}$, Current Contents ${ }^{\circledR} /$ Clinical Medicine, $^{2}$
Journal Citation Reports/Science Edition, EMBase, Scopus and the Elsevier Bibliographic databases. The manuscript management system is completely online and includes a very quick and fair peer-review system, which is all easy to use. Visit http://www.dovepress.com/ testimonials.php to read real quotes from published authors. 